SIMULACIÓN Y EVALUACIÓN DE UNA VOLADURA DE PRODUCCIÓN EN UNA CANTERA MEDIANTE ANÁLISIS CON EL SOFTWARE JKSIMBLAST, PERFILOMETRÍA LÁSER 2D Y SISMÓGRAFOS.

Antonio Morato, Benjamín Cebrián. 


\title{
Simulación y evaluación de una voladura de producción en una cantera mediante análisis con el software JKSimBlast, perfilometría láser 2D y sismógrafos.
}

\section{Simulation and evaluation of a production blasting in a quarry through analysis with JKSimBlast software, 2D laser profilometry and seismographs.}

\author{
Antonio Morato Medina ${ }^{1}$, Benjamín Cebrián Romo ${ }^{2}$ \\ ${ }^{1}$ Universidad Estatal Península de Santa Elena (UPSE) \\ Campus La Libertad, vía principal Santa Elena - La Libertad \\ La Libertad-Ecuador \\ amorato@upse.edu.ec \\ ${ }^{2}$ Blast Consult S.L. \\ Calle José Fentanés número 42, Madrid \\ Madrid-España \\ benjamin.cebrian@blast-consult.com
}

\section{Resumen}

En la cantera de árido de estudio se extrae roca caliza para su explotación como material de construcción. La función principal de las voladuras de producción es obtener mediante el uso de explosivos civiles, un tamaño de bloque adecuado para la planta de trituración, conseguir un frente de talud estable, bien marcado y rectilíneo para garantizar la estabilidad de la explotación y minimizar las vibraciones en las estructuras y zonas de interés circundantes. Para ello se define una voladura tipo en la que se simulan y evalúan los principales parámetros de diseño mediante el uso del software JKSimBlast, un perfilómetro $2 D$ y dos sismógrafos, buscando conciliar una producción óptima con un nivel mínimo de vibraciones en las zonas a monitorear.

Palabras clave: voladura, vibraciones, sismógrafo, JKSimBlast, cantera.

\begin{abstract}
At the studied quarry, limestone is extracted for its exploitation as construction material. The main functions of production blasts are to obtain, by using civil explosives, a suitable block size for the crushing plant, to achieve a stable, well-marked and rectilinear slope, to guarantee the stability of the operation and to minimize the vibrations in the surrounding structures and areas of interest. For this, a type blast is defined in which the main design parameters are simulated and evaluated using JKSimBlast software, a $2 D$ profiler and two seismographs, seeking to reconcile optimal production with a minimum level of vibration in the areas to be monitored.
\end{abstract}

Keywords: blasting, vibrations, seismograph, JKSimBlast, quarry. 


\section{Introducción}

En este estudio se ha pretendido evaluar el diseño de una voladura tipo de producción en una cantera de calizas aplicando diversas técnicas en las fases de diseño de la voladura y en la de control de resultados.

Para ello, se ha combinado un diseño con un software minero de simulación de voladuras, el JKSimBlast, que permite hacer una evaluación de diversos parámetros de la voladura tales como carga operante real, patrón de desplazamiento, vibraciones, niveles de energía en planos verticales y horizontales, etc., con la perfilometría láser de todos los barrenos de la primera fila de la voladura durante el proceso de marcado de la misma. Asimismo, se controló la calidad de la perforación, comparando las longitudes teóricas diseñadas con la que realmente se perforó para cada barreno.

Todo ello se enfoca a conseguir un patrón de voladuras que tenga optimizado el consumo de explosivos y genere una fragmentación óptima, y además no cause proyecciones horizontales, verticales y/o unas vibraciones altas.

A nivel general, el fenómeno de las vibraciones y el efecto que producen en estructuras y en las canteras o minas ha sido estudiado y de hecho todavía es objeto de numerosos análisis. ${ }^{[1-5]}$

El software elegido para la simulación de los resultados de la voladura ha sido utilizado también por diversos autores en varios trabajos. ${ }^{[6-7]}$

En la cantera elegida se han llevado a cabo previamente otros estudios para modelizar algunos parámetros en otro tipo de voladuras. ${ }^{\text {[8-10] }}$

El objetivo que se persigue con la evaluación del patrón de voladura que se va a implementar es conseguir una pila de voladura con una fragmentación adecuada para la planta de trituración, su procesamiento en molinos primarios y secundarios de producción de árido (grava y arena), así como eliminar las proyecciones horizontales y verticales y minimizar la vibración.

Para ello, es imprescindible y recomendable la combinación de técnicas de simulación y control de implementación que incluyen el uso del software más moderno en gestión de voladuras, perfilometría láser en dos dimensiones para implementar correctamente la primera fila de barrenos y un exhaustivo control de la perforación y el nivel de vibraciones.

\section{Materiales y métodos}

Para todo el proceso de diseño de la voladura y posterior simulación y análisis de los datos, se utilizan diferentes equipos.

Para obtener unos resultados óptimos es fundamental que la implementación del diseño sea correcta, y sobre todo, garantizar que la fila crítica, que es la primera, esté correctamente diseñada y marcada en campo: Con piedra (burden) ni excesiva ni deficiente, lo cual condiciona el confinamiento del explosivo.

Para conseguir esto, se realiza una perfilometría láser de cada barreno de la primera fila, que va a permitir su correcta localización para mantener los valores de piedra que se diseñan.

Una correcta implementación de la primera fila sin excesos ni defectos de piedra garantiza mantener los niveles de vibración y las proyecciones horizontales bajo control.

Para la realización de la simulación de la voladura se utiliza el software JKSimBlast, concretamente el módulo 2DBench. Este programa va a evaluar tanto el nivel energético alcanzado por el explosivo en los niveles que se consideran de interés, como hacer una predicción de la dirección de salida de la voladura en función de la secuenciación.

Un barreno tipo se carga en condiciones secas con $5 \mathrm{~kg}$ de dinamita gelatinosa Eurodyn 65/500 (dos cartuchos) y $142 \mathrm{~kg}$ de Exan (con una carga lineal media de $7.6 \mathrm{Kg} / \mathrm{m}$ ), tomando un retacado de $3 \mathrm{~m}$.

En los barrenos con agua se utiliza adicionalmente cordón detonante de $12 \mathrm{~g} / \mathrm{m}$ para asegurar la detonación, cargando además con explosivo encartuchado hasta superar el nivel del agua.

Para la configuración de tiempos se realiza una conexión en diagonal utilizando para la línea maestra conectores de superficie de $25 \mathrm{~ms}$ y para las diagonales conectores de superficie de $42 \mathrm{~ms}$, como se ve en la figura 1.

El ángulo de perforación es de $15^{\circ}$, excepto dos barrenos laterales que se marcan a $10^{\circ}$. Teniendo en cuenta la conexión, las líneas de isotiempo de la voladura quedan definidas tal y como se muestra en la figura $\mathrm{n}^{\circ} 1$, siendo la dirección teórica de salida de la voladura perpendicular a ellas. La distribución de energía del explosivo se consigue mediante una malla adecuada, perforada al tresbolillo para una mejor distribución energética y un nivel de retacado acorde al diámetro de perforación y a la geología y geotecnia del macizo rocoso a volar.

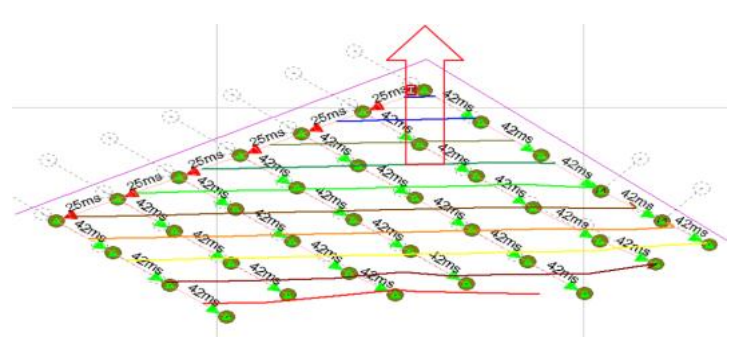

Figura 1. Secuenciación y líneas de isotiempos de la voladura.

En este caso, la voladura fue perforada siguiendo un 
patrón de malla de 4.2 x $4.6 \mathrm{~m}$, con 3 metros de retacado. El diámetro de perforación fue de $110 \mathrm{~mm}$.

A continuación, se ilustran diferentes cortes horizontales de distribución de energía del explosivo utilizado (figura 2).

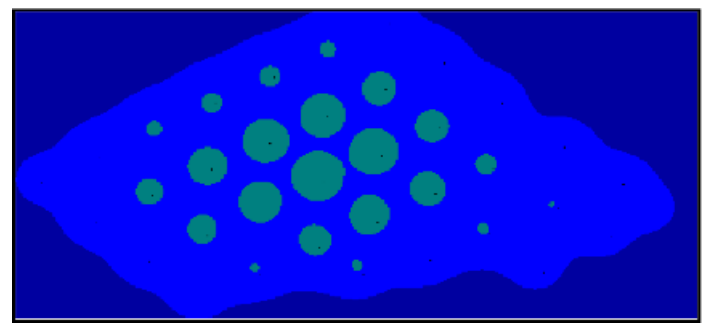

a)

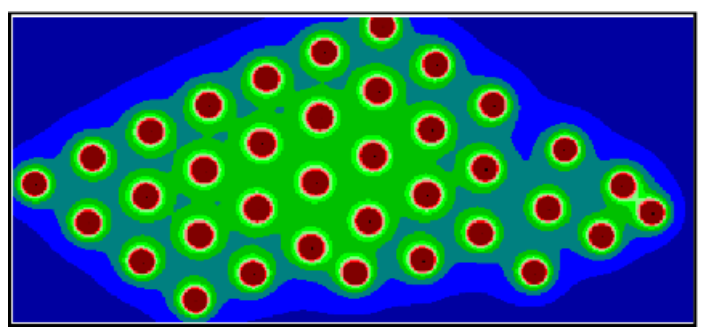

b)

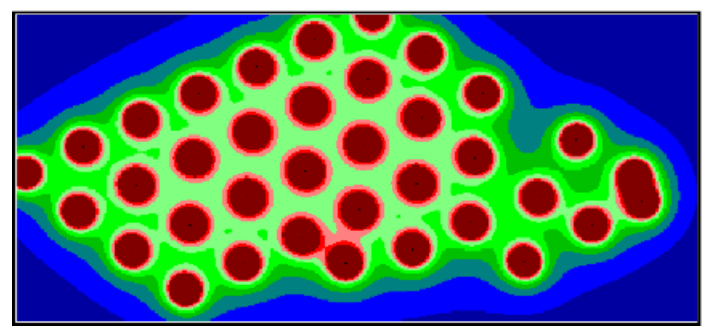

c)

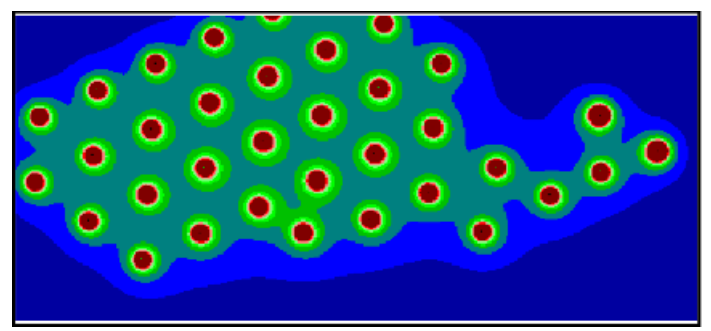

d)

Figura 2. Cortes energéticos horizontales. a) A nivel de retacado ( $1.5 \mathrm{~m}$ de profundidad). b) Contacto retacado explosivo ( 3 de profundidad). c) Nivel de explosivo ( 9 de profundidad). d) Nivel de pie ( $22 \mathrm{~m}$ de profundidad).

La escala utilizada en megajulios/tonelada se muestra en la figura 3.

Para el control de vibraciones se utilizó un sismógrafo White modelo Miniseis, con número de serie 121, para la monitorización ambiental de vibraciones. Se colocó a $550 \mathrm{~m}$ de la voladura. Con este sismógrafo se pretende evaluar el posible daño a la estructura a proteger que pueda causar la voladura.

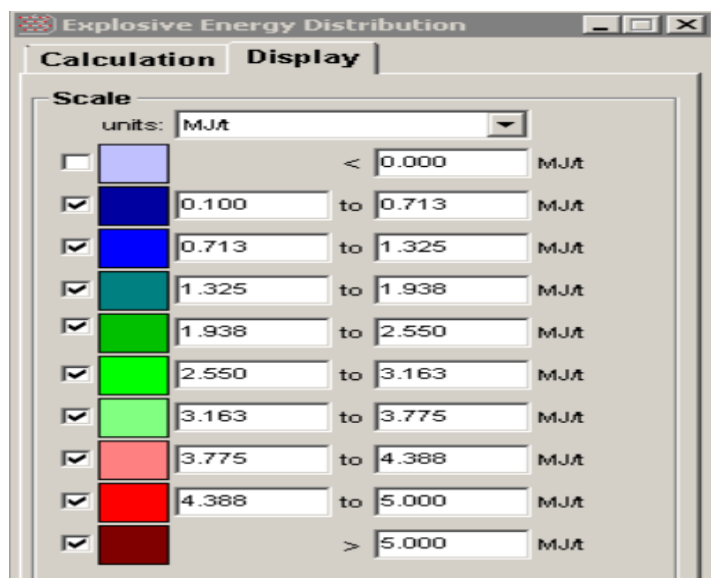

Figura 3. Escala de energía de la voladura.

Para ello se aplica la Norma UNE 22.381-93 de Control de Vibraciones Producidas por Voladuras [11], en concreto su punto 5 "Criterio de prevención de daños", que relaciona la máxima velocidad pico admisible $(\mathrm{mm} / \mathrm{s})$ en función de la frecuencia principal $(\mathrm{Hz})$ y del tipo de estructura a proteger.

Adicionalmente, para el control de daños a taludes, se utilizó un sismógrafo Instantel Minimate Plus con número de serie BE 18051, que se colocó a $60 \mathrm{~m}$ de la voladura, a pie de talud, para evaluar los efectos de la vibración en el material de esta zona.

Se sigue el criterio de Bauer y Calder ${ }^{[12]}$ aplicado a taludes, teniendo como valor límite para daños 250 $\mathrm{mm} / \mathrm{s}$.

\section{Resultados}

Las evaluaciones de resultados se centraron en una inspección de la pila de voladura obtenida, revisando el despegue del talud, la fragmentación y el nivel de vibración alcanzado en los puntos de estudio, así como la calidad de la perforación.

En primer lugar, previo al disparo y carga de la voladura se realizó un estudio de la calidad de perforación, que se presenta en las figuras 4 y 5 .

Se toma como valor excelente de perforación un intervalo de desvío sobre la longitud proyectada de $0,1 \mathrm{~m}$. Como valores aceptables se toman aquellos donde la tasa de desvío sobre la longitud proyectada comprenda valores entre 0,1 y $0,3 \mathrm{~m}$. Como valores malos se toman aquellos que presenten una desviación sobre la longitud proyectada de entre 0,3 y $0,5 \mathrm{~m}$. Y como valores muy malos aquellos que presenten un desvío superior a 0,5 metros sobre la longitud proyectada. 


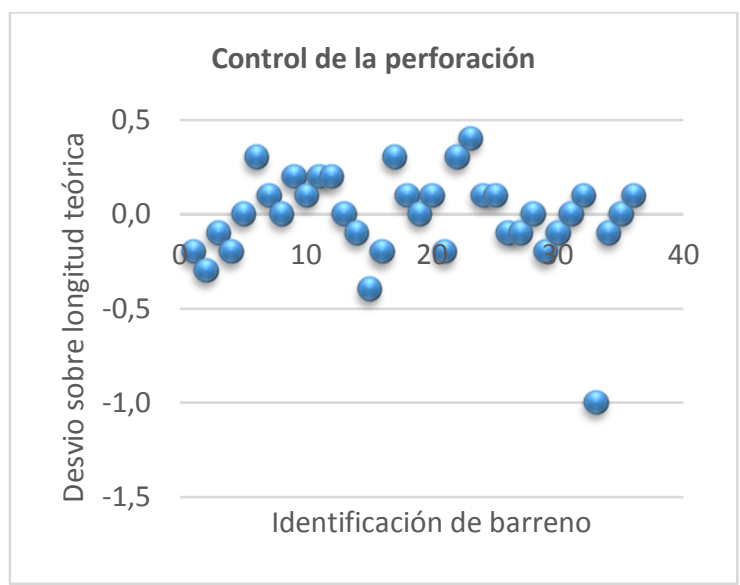

Figura 4. Control de la perforación.

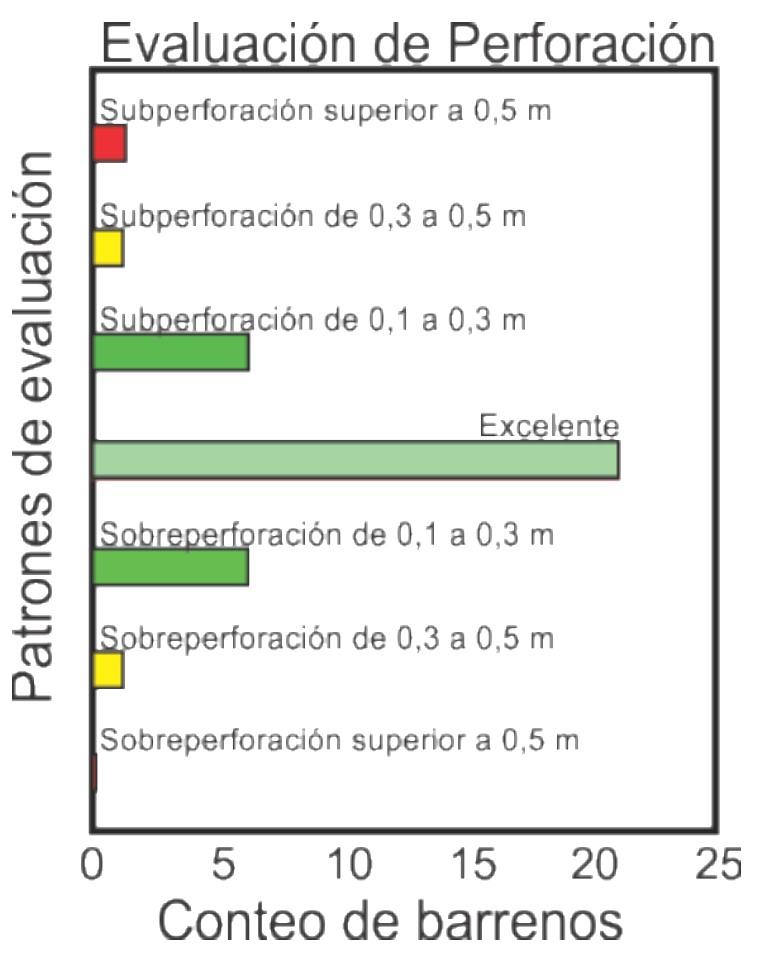

Figura 5. Evaluación de la calidad de perforación.

En cuanto a la fragmentación y desplazamiento, se obtuvo una pila de voladura compacta (fotografía 1), apta para la carga tanto por retroexcavadora como por pala frontal y homogéneamente fragmentada, con un P80 inferior a los $700 \mathrm{~mm}$ de diámetro que acepta como máximo la planta de trituración de la cantera.

El talud resultante quedó en óptimas condiciones, con las cañas de los barrenos marcadas, lo que indica que no hubo un exceso de confinamiento en ninguno de los tiros.

En las fotografías se observa que los gases generados por el explosivo trabajaron correctamente $\mathrm{y}$ no se produjeron proyecciones verticales ni horizontales.

Se observa la aparición de humos anaranjados (óxidos de nitrógeno), que indican una detonación defectuosa de Exan (Anfo) en uno de los barrenos que tenía agua (fotografía 2).

Se formaron algunos sobre-tamaños en la zona superior de la voladura, y un bloque preformado en el frente, no logró fragmentar bien. (fotografías 3 y 4). Los tamaños gruesos presentes en la parte superior del retacado son causados por una longitud de taco alta ( $3 \mathrm{~m}$ o 26 diámetros de carga), que garantiza por un lado el control de proyecciones verticales y el aprovechamiento íntegro de la energía explosiva y, por otro, permite la generación de un porcentaje de escollera para venta directa.

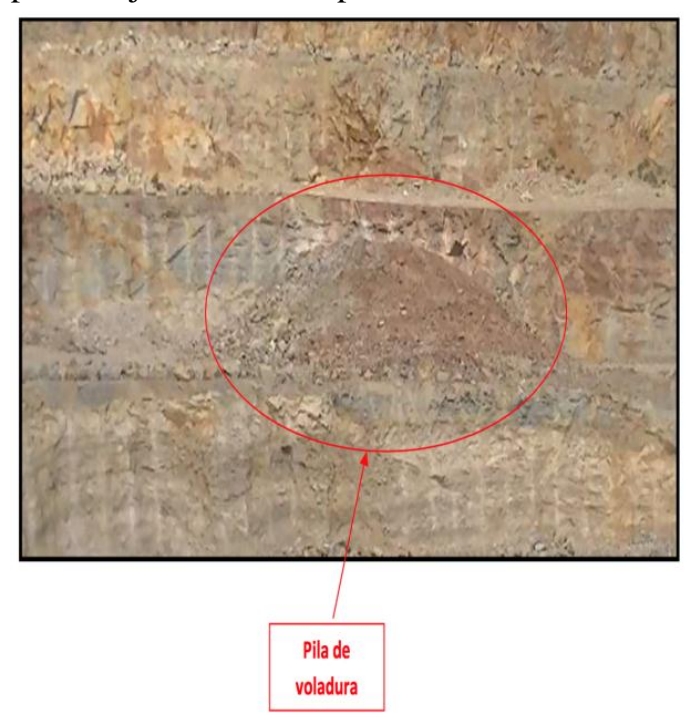

Fotografía 1. Pila de voladura.

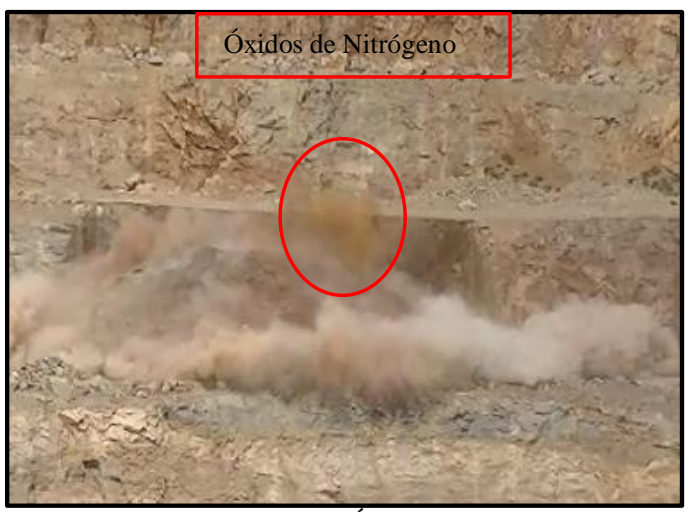

Fotografía 2. Presencia de Óxidos de Nitrógeno. 

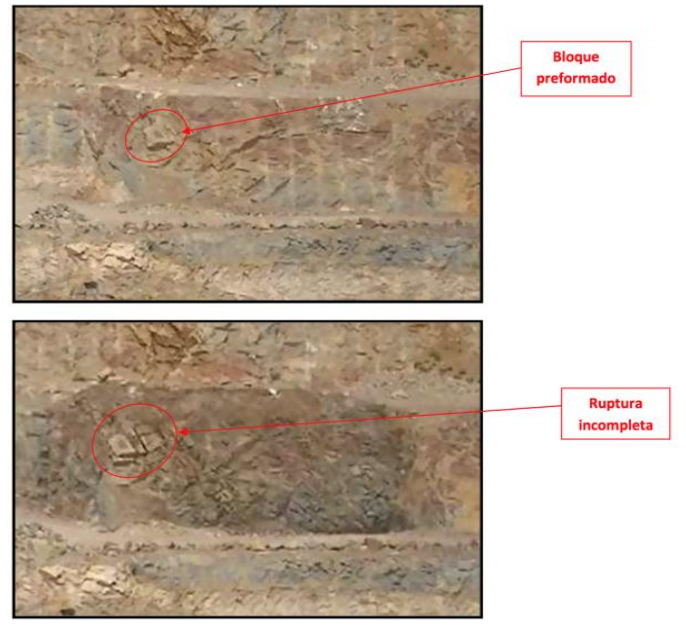

Fotografías 3 y 4 . Ruptura incompleta de bloque preformado.

Los dos sismógrafos que se colocaron para controlar y evaluar el nivel de vibraciones se situaron en las siguientes localizaciones en el interior de la cantera (fotografía 5):

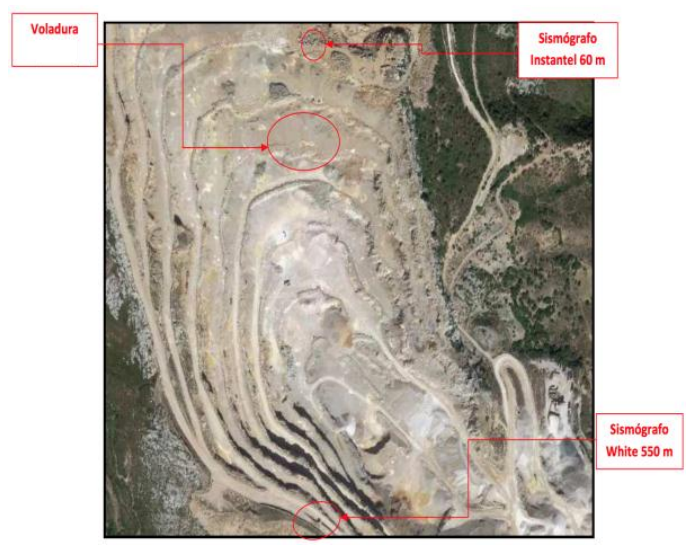

Fotografía 5. Colocación de sismógrafos respecto a la voladura.

Los valores de vibración registrados por cada uno de ellos se exponen en la siguiente tabla:

Tabla 1: Registro de vibraciones.

\begin{tabular}{|c|c|c|c|c|c|c|c|c|c|}
\hline \multicolumn{10}{|c|}{ VIBRACIONES } \\
\hline \multirow{2}{*}{ SISMOGRaF0 } & \multirow{2}{*}{ Sirvacón } & \multirow{2}{*}{ DistancaA } & \multirow{2}{*}{ Freq(Hz) } & \multicolumn{3}{|c|}{$p p V(m m / s)$} & \multicolumn{3}{|c|}{ 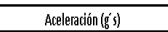 } \\
\hline & & & & Yetitial & Longitudina & Iransueral & Vertial & Longitudina & Transversal \\
\hline White & Báscula & 550 & 11.1 & 2.54 & 3.302 & 1.778 & 0.027 & 0.027 & 0.013 \\
\hline Instantel & Zona mármol patio 5 & 60 & 23.5 & 22.2 & 34 & 28.2 & 0.848 & 1.06 & 0.848 \\
\hline
\end{tabular}

A continuación se muestran los sismogramas obtenidos de cada uno de los sismógrafos. Se presentan en las figuras 6,7 y 8 .

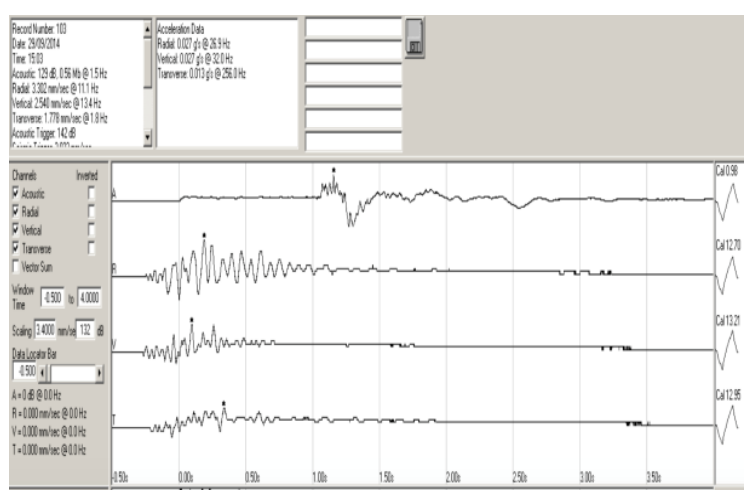

Figura 6. Registro de Sismógrafo White.

$\begin{array}{lrrrl} & \text { Tran } & \text { Vert } & \text { Long } & \\ \text { PPV } & 28.2 & 22.2 & 34.0 & \mathrm{~mm} / \mathrm{s} \\ \text { PPV (Ponderado) } & 27.2 & 18.3 & 32.0 & \mathrm{~mm} / \mathrm{s} \\ \text { PPV } & 80.0 & 77.9 & 81.6 & \mathrm{~dB} \\ \text { Frecuencia ZC } & 22.3 & 37.2 & 23.5 & \mathrm{~Hz} \\ \text { Tiempo (Rel. al Disparador) } & 0.256 & 0.167 & 0.237 & \mathrm{seg} \\ \text { Aceleracion del Pico } & 0.848 & 0.848 & 1.06 & \mathrm{~g} \\ \text { Desplazamiento del Pico } & 0.188 & 0.0963 & 0.214 & \mathrm{~mm} \\ \text { Chequeo de Sensores } & \text { Paso } & \text { Paso } & \text { Paso } & \\ \quad \text { Frequency } & 7.4 & 7.5 & 7.4 & \mathrm{~Hz} \\ \quad \text { Overswing Ratio } & 3.6 & 3.7 & 3.6 & \end{array}$

Pico Vector Suma $41.9 \mathrm{~mm} / \mathrm{s}$ en $0.237 \mathrm{seg}$

Figura 7. Registro numérico de sismógrafo Instantel.

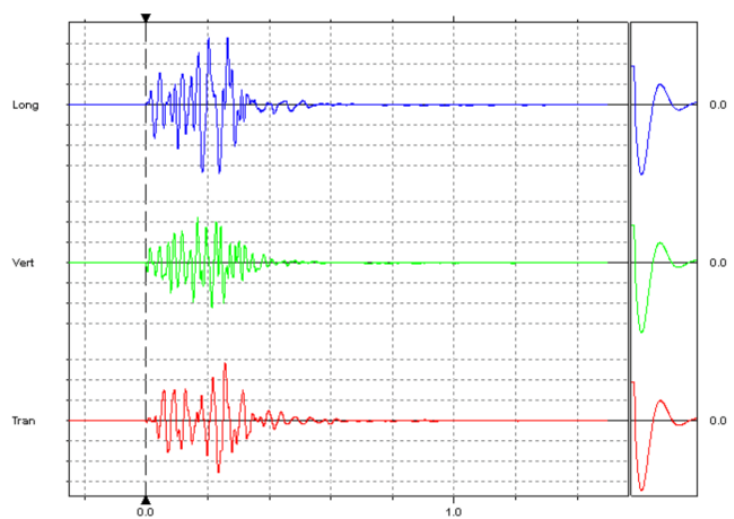

Figura 8. Registro de Sismógrafo Instantel.

\section{Discusión de los resultados}

Tras la revisión de los resultados obtenidos, el patrón de voladura se considera óptimo en términos relacionados con la producción. La fragmentación de la roca es la deseada y el desplazamiento y geometría de la pila de voladura la hace compatible para la carga con la maquinaria de la cantera.

Solamente 3 barrenos se desvían del estándar de perforación implantado, por caída de material suelto. Se consideró necesario reperforar uno de ellos, pues tenía un metro menos de longitud sobre lo proyectado.

La longitud de taco ha permitido tanto el correcto confinamiento del explosivo, pues no se observa en las imágenes que haya proyectado verticalmente ningún barreno, como la aparición de algunos bloques de escollera, que era uno de los objetivos secundarios que se perseguía con la voladura. 
La perfilometría laser de la primera fila de la voladura ha permitido un confinamiento correcto de la voladura y el que no aparezcan proyecciones horizontales. El explosivo, tal y como se observa en las imágenes, ha trabajado de forma homogénea a lo largo de toda la voladura.

Respecto a las vibraciones, el sismógrafo White Miniseis permite un estudio de prevención de daños adaptado por componente de onda (vertical, radial y transversal) al criterio de la Norma UNE 22381 de Control de daños producidos por Voladuras ${ }^{[11]}$.

El software que lo gestiona puede mostrar graficadas la velocidad de partícula en $\mathrm{mm} / \mathrm{s}$ en el eje Y y la frecuencia de vibración en $\mathrm{Hz}$ en el eje X. Así mismo representa los umbrales que no se pueden superar por el grueso de puntos de vibración para estructuras tipo I, II y III (las más sensibles), como se observa en las figuras 9, 10 y 11.

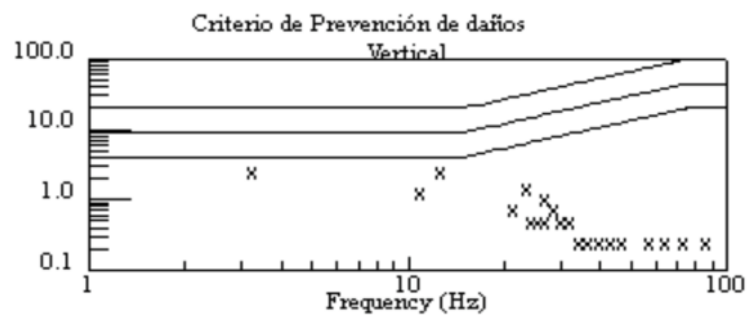

Figura 9. Criterio de prevención de daños componente vertical.

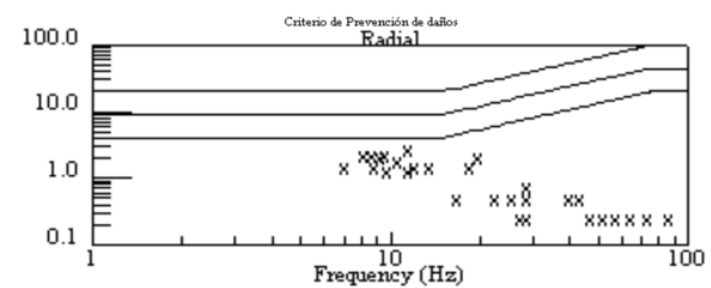

Figura 10. Criterio de prevención de daños componente radial.

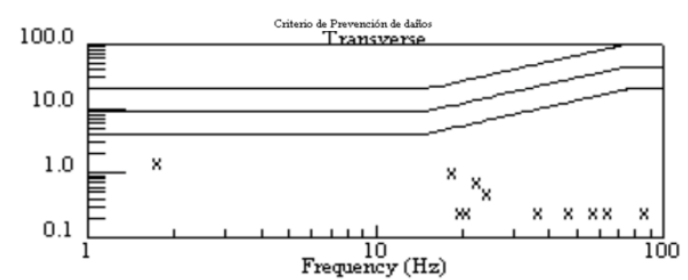

Figura 11. Criterio de prevención de daños componente transversal.

Ninguno de los registros del sismógrafo en cualquiera de las tres componentes supera los niveles de estructuras tipo III, que son los más restrictivos, y que corresponden a la línea inferior de los gráficos, por lo que se puede afirmar que la voladura no causa daños a ningún tipo de estructuras que estén alejadas de ella una distancia igual o superior a $550 \mathrm{~m}$ a los que se colocó el sismógrafo.

En cuanto a la prevención de daños a taludes, se sigue el criterio de Bauer y Calder (1971) ${ }^{[12]}$ aplicado a taludes, teniendo como valor límite para daños de $250 \mathrm{~mm} / \mathrm{s}$.

\section{A.Morato/B. Cebrián}

\begin{tabular}{|c|l|}
\hline $\begin{array}{c}\text { VELOCIDAD DE } \\
\text { PARTICULA (cm/s) }\end{array}$ & \multicolumn{1}{|c|}{ DANOS PREVISIBLES } \\
\hline \hline 25 & - No hay peligro en roca sana \\
25.60 & - Puede aparecer descostramiento de lajas por rotura a tracción \\
$60 \cdot 250$ & - Grandes roturas por traccion y algunas grietas radiales \\
$>250$ & - Agrietamiento total del macizo rocoso \\
\hline
\end{tabular}

Figura 11. Criterio de prevención de daños a taludes. Tomada de López ${ }^{[13]}$

Los niveles de vibración no superaron este umbral a una distancia de $60 \mathrm{~m}$, por lo que no se esperan daños a los taludes adyacentes debido a las vibraciones generadas por la voladura.

\section{Conclusiones}

La implementación de métodos de simulación de voladuras con software específico, control en campo mediante evaluación de la perforación y perfilometría de taludes, así como la monitorización de vibraciones, se antoja fundamental para valorar, comprender y mejorar el comportamiento de una voladura de producción en una cantera de áridos. La integración de estos métodos y los resultados obtenidos han permitido obtener y medir unos resultados adecuados en función de los objetivos de fragmentación y control de proyecciones y vibraciones que se perseguían.

El tener bajo control todos los parámetros ha permitido alcanzar los objetivos de producción propuestos, así como una mejora en materia de seguridad al eliminar completamente la ocurrencia de proyecciones.

Con los datos obtenidos, se puede también afirmar que las vibraciones producidas por la voladura en ningún caso han afectado ni a la estructura que tenía que protegerse ni a los taludes de la explotación.

\section{Bibliografía}

[1] Yang, J., Lu, W., Jiang, Q., Yao, C., Zhou, C. 2016. Frequency comparison of blast-induced vibration per delay for the full-face millisecond delay blasting in underground opening excavation.

Tunnelling and Underground Space Technology, Volumen 51, pp 189-201.

[2] Trigueros, E., Cánovas, M., Muñoz, J., Cospedal, J. 2017. A methodology based on geomechanical and geophysical techniques to avoid ornamental stone damage caused by blast-induced ground vibrations. International Journal of Rock Mechanics and Mining Sciences, Volumen 93, pp 196-200.

[3] Singh, P., Roy, M., Paswan, R., Sarim, Md., Kumar, S., Rakesh Ranjan Jha. 2016. Rock fragmentation control in opencast blasting. Journal of Rock Mechanics and Geotechnical Engineering, Volumen 8, pp 225-237. 
[4] Tripathy, G., Shirke, R., Kudale, M. 2016. Safety of engineered structures against blast vibrations: A case study. Journal of Rock Mechanics and Geotechnical Engineering, Volumen 8, pp 248-255. [5] Lee, J., Ahn, S., Sagong, M. 2016. Attenuation of blast vibration in tunneling using a pre-cut discontinuity. Tunnelling and Underground Space Technology, Volumen 52, pp 30-37.

[6]. Onederra, I. \& Esen, S. 2003. Selection of interhole and inter-row timing for Surface blasting-an aproach based on burden relief analysis. Explosive and Blasting Technique. Edited by R. Holmberg. Chapter 33. pp 269-275.

[7]Villaescusa, E. 2014. Geotechnical Design for Sublevel Open Stoping. Edited by CRC Press. 541 pág.

[8] Maqueda, S. 2008. Relación entre el control de parámetros de voladuras adecuados y disponibilidad de planta de tratamiento. Ingeopres $n^{\circ} 171$, pp 38-39.
[9] Cebrian, B. 2005. Accesorios especiales de voladura: bolsas autoinflables (Baif) Técnicas especiales de voladuras. Ingeopres $n^{\circ} 142$, pp 20-22 [10] Orive, J., Sáez, J., Cebrián, B. Optimización de una voladura de escollera. Ingeopres $n^{\circ} 182$, pp 3638.

[11] UNE 22.381. 1993. Control de Vibraciones Producidas por Voladuras. AENOR, abril de 1993.

[12] Bauer, A. \& Calder, P. 1971. The Influence and Evaluation of Blasting on Stability. In Stability in Open Pit Mining, Proc. 1st International Conference on Stability in Open Pit Mining. New York: Society of Mining Engineers, A.I.M.E, pp. 83-94.

[13] López, C. 2003. Manual de Perforación y Voladura de Rocas. Editorial: Carlos López Jimeno. 778 pág. 